\title{
Persistent primitive hypoglossal artery associated with lower basilar artery aneurysm: Diagnosis and clinical implications
}

\author{
Sunil V. Furtado, Kalyan Reddy ${ }^{1}$, N ilesh Khandelwal ${ }^{1}$, A. S. Hegde \\ Departments of Neurosurgery and ${ }^{1}$ Radiology, Sri Satya Sai Institute of Higher Medical Sciences, Bangalore, India
}

\author{
Address for correspondence: \\ Dr. Sunil V. Furtado, \\ Department of Neurosurgery, \\ Sri Satya Sai Institute of Higher \\ Medical Sciences, EPIP Area, \\ Whitefield, Bangalore - 560 066, \\ India. \\ E-mail: sunilvf@gmail.com
}

DOI: $10.4103 / 0028-3886.48809$

\begin{abstract}
An elderly male was evaluated for subarachnoid hemorrhage and found to have a persistent primitive hypoglossal artery with its associated abnormal posterior fossa circulation and a lower basilar artery aneurysm. Angiography findings are described and are corroborated with computed tomography and magnetic resonance imaging. Its surgical and clinical relevance are discussed.
\end{abstract}

Key words: Basilar artery, hypoglossal, intracranial aneurysm, subarachnoid hemorrhage

\section{Introduction}

During embryogenesis, anastomoses are formed between the carotid artery and the basilar or the vertebral artery, which regress with maturity of the vertebral artery. A persistent primitive hypoglossal artery (PHA), which is a segmental vessel, is a rare anomaly. It usually represents an incidental finding in cerebral angiograms and is associated with unique anomalies of posterior fossa circulation. This embryonic remnant is clinically significant when associated with aneurysm or atherosclerotic disease of the posterior circulation. ${ }^{[1]}$ About 30 cases of posterior circulation aneurysms associated with PHA are documented in English literature.

\section{Case Report}

A 65-year-old male with no significant past medical history presented with severe headache, vomiting and loss of consciousness. A plain computed tomogram (CT) scan done at the referring hospital on the fourth day of ictus revealed Fisher Grade II bleed with blood in the prepontine, ambient cisterns and sylvian fissures [Figure 1]. There was no intraventricular blood or hydrocephalus. He was started on nimodepine $(60 \mathrm{mg}$ 4 hourly), dexamethasone $4 \mathrm{mg} 8$ hourly, promethazine $25 \mathrm{mg} 8$ hourly, phenytoin $100 \mathrm{mg} 8$ hourly and kept well hydrated. Two weeks after referral to our hospital he had improved and was in Hunt and Hess Grade I, with no significant neurological signs.

A digital substraction angiogram (DSA) revealed a PHA on the left side, arising from the cervical internal carotid

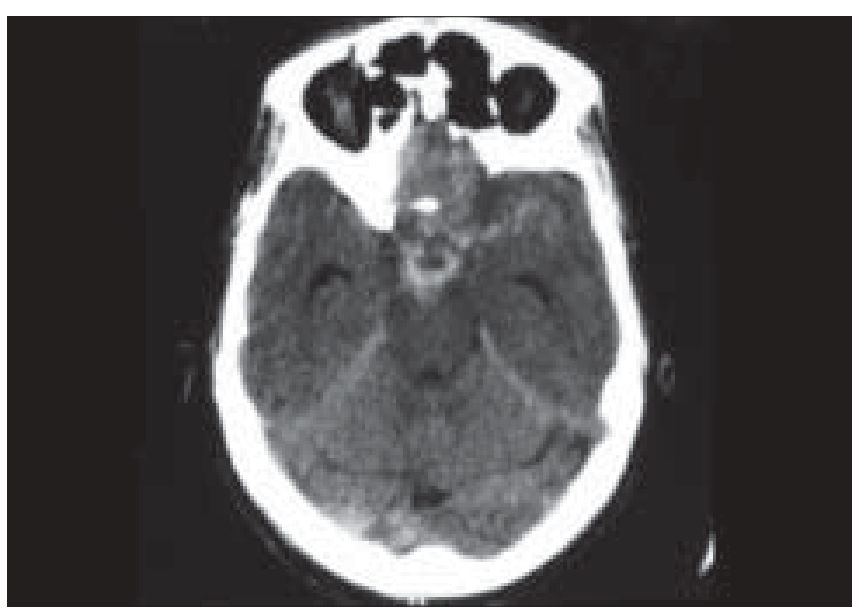

Figure 1: CT scan on fourth day after ictus demonstrating SAH in the prepontine cistern and sylvian fissure 
artery (ICA) at C2-3 level [Figure 2a, Figure 2b]. It was seen on left ICA injection and entered the posterior fossa at the region of the hypoglossal canal and joining the basilar artery (BA) at the lower clivus [Figure 2a, Figure 2b]. The right vertebral artery (VA) appeared hypoplastic [Figure 3b]. Both posterior communicating arteries (PCOM) were not visualized on right, left ICA and VA injections. There was a narrow-necked multi- lobulated saccular aneurysm in the lower BA segment, $9 \times 5 \times 11 \mathrm{~mm}$ in size, projecting horizontally to the right and filling on left ICA and left VA injection. Rest of the intracranial circulation was normal.

The left hypoglossal canal was wider compared to the right [Figure 4a]. Magnetic resonance (MR) 3D-TOF SPGR sequence showed a large vessel entering the posterior fossa through the left hypoglossal canal [Figure 4b]. MR imaging (MRI) and MR angiography (MRA) were done to qualify the anatomical relationship between the abnormal posterior circulation, aneurysm,

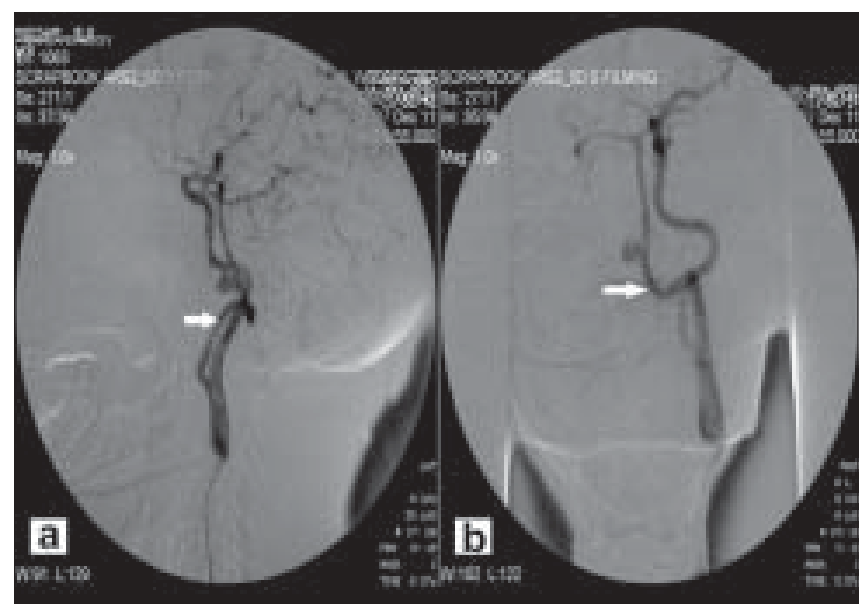

Figure 2: DSA demonstrating filling of basilar artery and lower basilar aneurysm on left ICA injection through the left persistent hypoglossal artery (white arrow) (a) lateral view (b) oblique view

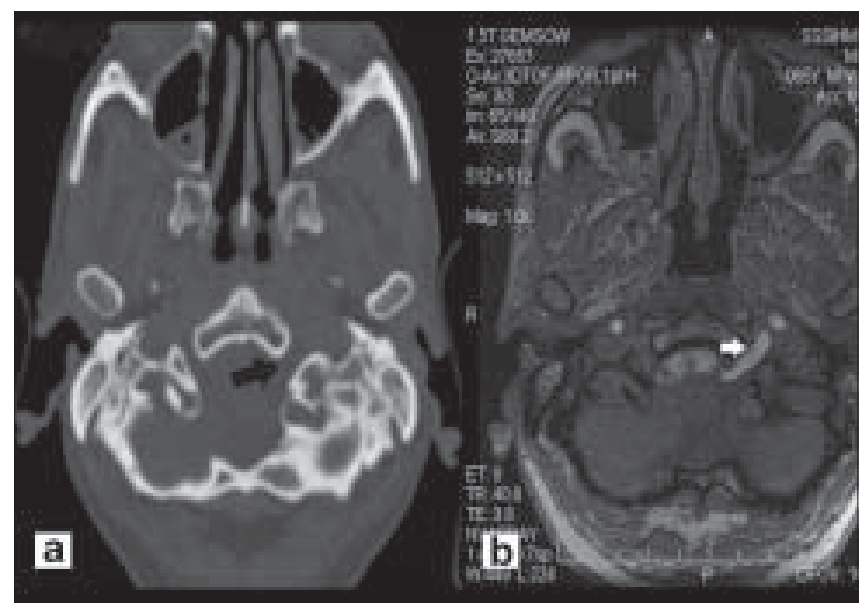

Figure 4: (a) Dark arrow pointing to an enlarged left hypoglossal foramen (b) Left persistent hypoglossal artery seen (white arrow) in the hypoglossal canal on 3D-TOF SPGR sequence brainstem and bony posterior fossa structures.

The patient underwent a right lateral suboccipital craniectomy. Anesthesia was maintained with a combination of isofluorane, propofol, fentanyl and vecuronium. He was administered $1.5 \mathrm{~g}$ methylprednisolone at craniotomy and $0.5 \mathrm{~g} / \mathrm{kg}$ body weight of mannitol at durotomy for cerebral protection. Cerebellar retraction exposed petrous dura and lower cranial nerves. The aneurysm was adherent to dura on lower clivus and was approached between the lower cranial nerves. The aneurysm was distal to the formation of the BA, from the union of the PHA and left VA and above the left posterior inferior cerebellar artery (PICA). There was no evidence of vasospasm. The aneurysm was dissected off the dura and clipped uneventfully, after visualizing the BA, proximal and distal to the aneurysm [Figure 5a, Figure 5b]. Mean blood pressure was maintained around 60-65 mm Hg during dissection and clipping. Aneurysm fundus

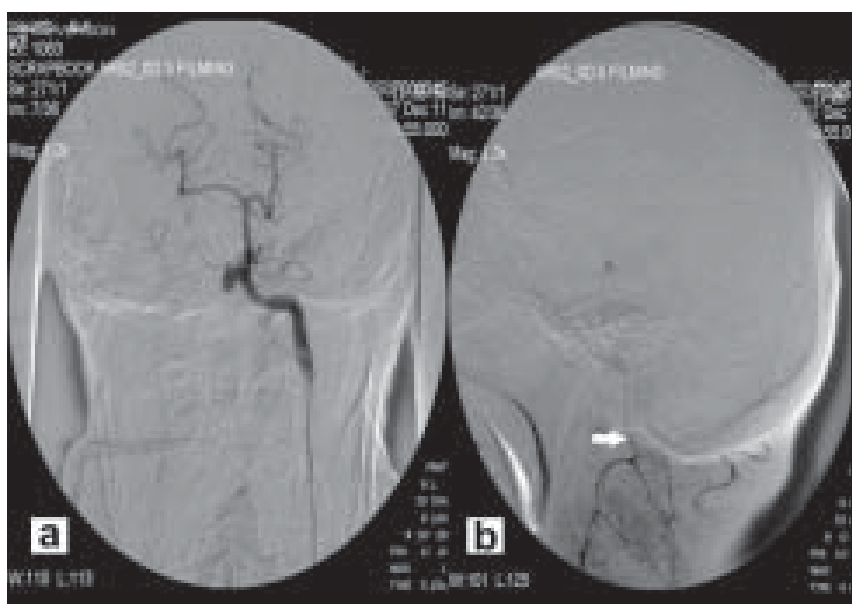

Figure 3: (a) Left vertebral artery injection demonstrating lower basilar aneurysm (b) Hypoplastic right vertebral artery (white arrow) on right vertebral artery injection

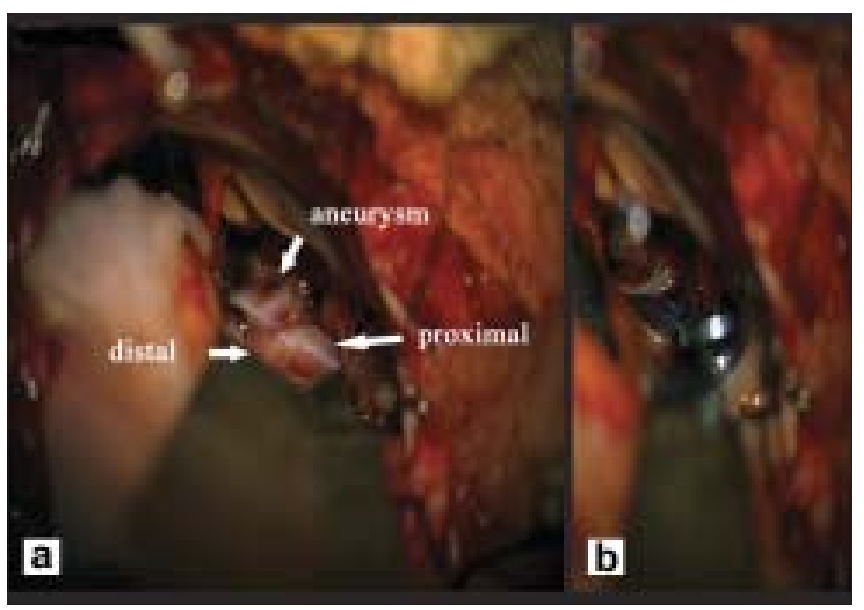

Figure 5: (a) Cerebellum has been retracted to show the neck of a multilobed aneurysm with lower basilar artery proximal and distal to its neck (b) Clip in situ across aneurysm neck 
was not excised. Apprehensions existed regarding the visualization of BA and aneurysmal neck in the clival midline due to subarachnoid inflammation. However, drainage of cisterna magna cerebrospinal fluid and a reverse Trendelenberg position allowed retraction of a lax cerebellum and proper visualization. Patient had an uneventful postoperative period and did not have any lower cranial nerve deficits or intrinsic brainstem signs. He was kept on triple-H regimen for a week after surgery along with Nimodepine.

\section{Discussion}

PHA, with an incidence of $0.03-0.26 \%$ of cerebral angiograms is the second most common carotid basilar anastomosis, after persistent trigeminal artery. ${ }^{[1]}$ Its presence can be explained by the carotid basilar anastomosis which develops between the anterior and posterior circulation in the $4-5 \mathrm{~mm}$ embryo, as the vertebral arteries are not yet formed. ${ }^{[2]}$ The segmental anastomotic channels like the trigeminal, otic and hypoglossal are named after the nerve they accompany. Failure of involution of segmental anastomosis beyond the 40th day, when the PCOM are well developed, leads to their sporadic presence. ${ }^{[1]}$

The anomaly, first described in 1889, is most often demonstrated during angiography for sub-arachnoid hemorrhage (SAH), arteriovenous malformations, ischemic cerebrovascular events and tumors. ${ }^{[2,3]} \mathrm{Lie}^{[4]}$ laid down four criteria for identification of PHA: a) it arises from the cervical part of the ICA at the C1-C2 level; b) it enters the posterior cranial fossa via the hypoglossal canal, c) basilar trunk must fill distal to the anomalous vessel and d) absent PCOM. ${ }^{[4]}$ However, recognition of the artery in noninvasive imaging is important. The artery widens the hypoglossal canal on CT and tomograms. ${ }^{[5]}$ The vessel can also be demonstrated entering through the hypoglossal canal and joining the BA using CT or MR angiography. These are acceptable diagnostic alternatives especially in incidentally detected cases. ${ }^{[6]}$ 3D-CT angiography and MRA may not detect multiple small unruptured aneurysms and aneurysms such as PCOM segment aneurysms. ${ }^{[6]}$ DSA remains the gold standard in the event of SAH, to localize the aneurysm and qualify the flow through abnormal posterior circulation and circle of Willis.

PHA has been variously associated with aneurysms of the BA, PICA and also PHA. The anomalous structure of the vessel wall of PHA exposes the basilar trunk to unusual hemodynamic stress and development of aneurysms. ${ }^{[1,7]}$ Kempe and Smith described a syndrome presenting with pain in the tonsillar fossa and posterior part of the tongue due to close association of the lower cranial nerves with PHA. ${ }^{[8]}$ Because of its origin from the
ICA and associated flow dynamics at the carotid bulb, atherosclerotic plaques can be expected. ${ }^{[9]}$ A typical distribution of emboli can lead to clinical dilemma. ${ }^{[1]}$

Associated hypoplasia of vertebral arteries and PCOM predisposes to a precarious posterior fossa circulation, making it susceptible to various surgical and thromboembolic insults. For all functional purposes, PHA may be the only dominant posterior fossa arterial supply. ${ }^{[7]}$ Careful understanding of the abnormal vasculature is important during aneurysm or skull-base surgery, carotid endarterectomy or endovascular intervention. Proper delineation of parent vessel proximal and distal to the aneurysm is important before clipping of aneurysms. In our case, this was necessitated because of a poor communicating supply across PCOM. Surgery is a formidable challenge in view of perilous circulation and site of aneurysm. Temporary clips proximal and distal to the aneurysm may be placed on the PHA, VA and BA respectively for a short period, with cerebral protection in the event of a rupture. Ligation of the PHA in such cases can have disastrous consequences. Aneurysm arising from PHA has been coiled using access through the residual segmental anomaly. ${ }^{[10]}$ Stent-assisted coiling is also a mode of treatment of these aneurysms. This modality provides a better chance of having a patent posterior circulation without concomitant surgical morbidity. Surgery can be considered in the event of complications or failure of endovascular treatment.

\section{References}

1. De Caro R, Parenti A, Munari PF. The persistent primitive hypoglossal artery: A rare anatomic variation with frequent clinical implications. Anat Anz 1995;177:193-8.

2. Jukic NB, Jelic M, Basic V, Jukic T, Vinter I. Persistent hypoglossal artery. J Anat 2001;198:315-6.

3. Venkatesh SK, Nangia S, Kathuria M, Phadke RV. Images: Persistent hypoglossal artery. Indian J Radiol Imaging 2001;11:29-30.

4. Chaljub G, Guinto FC Jr; Crow WN. Persistent hypoglossal artery: MRI and MRA findings. J Comput Assist Tomogr 1995;19:668-9.

5. Shapiro R. Enlargement of the hypoglossal canal in the presence of a persistent hypoglossal artery. Radiology 1979;133:395-6.

6. Oelerich M, Schuierer G. Primitive hypoglossal artery: Demonstration with digital subtraction-, MR-and CT angiography. Eur Radiol 1997;7:1492-4.

7. Kanai H, Nagai H, Wakabayashi S, Hashimoto N. A large aneurysm of the persistent primitive hypoglossal artery. Neurosurgery 1992;30:794-7.

8. Kempe LG, Smith DR. Trigeminal neuralgia, facial spasm, intermedius and glossopharyngeal neuralgia with persistent carotid basilar anastomosis. J Neurosurg 1969;31:445-51.

9. Wagner AL. Isolated stenosis of a persistent hypoglossal artery visualized at 3D CT angiography. A.JNR Am J Neuroradiol 2001;22:1613-4.

10. Bapuraj JR, Ojili V, Khandelwal N, Shanbhogue AK, Gupta SK. Basilar artery aneurysm treated with coil embolization via persistent primitive hypoglossal artery. Australas Radiol 2007;51:B340-3.

Accepted on 20-01-2009

Source of Support: Nil, Conflict of Interest: None declared. 